\title{
Intrinsic thermoelectric figure of merit of bulk compositional SiGe alloys: A first-principles study
}

\author{
Konstanze R. Hahn ${ }^{0},{ }^{*}$ Claudio Melis, Fabio Bernardini, and Luciano Colombo \\ Department of Physics, University of Cagliari, Cittadella Universitaria, 09042 Monserrato (CA), Italy
}

(Received 25 February 2021; revised 6 May 2021; accepted 20 May 2021; published 3 June 2021)

\begin{abstract}
A systematic study based on state-of-the-art first-principles calculations has been carried out to determine intrinsic thermoelectric properties in $n$ - and $p$-type SiGe alloys. Both electronic and thermal transport coefficients have been evaluated using the Boltzmann transport equation, applied on the electronic and phonon band structure, respectively. The Seebeck coefficient, electrical conductivity, and thermal conductivity have been analyzed focusing on the effect of carrier concentration, temperature, and alloy composition. The resulting figure of merit is highest for heavy doping and at elevated temperatures $(>1000 \mathrm{~K})$ in the SiGe alloy with Ge content from $50 \%$ to $80 \%$.
\end{abstract}

DOI: 10.1103/PhysRevMaterials.5.065403

\section{INTRODUCTION}

The rapid depletion of fossil fuels as a result of increasing and vast energy consumption should motivate a responsible use of energetic resources. At the same time, it is crucial to develop alternative and sustainable energy resources. In this framework, increased interest has been paid in recent years to the development of improved thermoelectric devices. Thermal energy in the form of waste heat is largely available in combustion-driven vehicles, food production, chemical processes, and in particular in power plants. In this respect, thermoelectric conversion provides the possibility of recovering a possibly large fraction of such waste heat and transforming it into electrical energy.

The overall efficiency of a thermoelectric device is governed by a complex interplay between materials issues and device characteristics [1]. As for the former ones, the dimensionless figure of merit $Z T$ summarizes the thermoelectric properties of a material: It is defined as $Z T=S^{2} \sigma / \kappa T$, where $S$ is the Seebeck coefficient, $\sigma$ is the electrical conductivity, and $\kappa$ is the thermal conductivity. Optimization of $Z T$ is far from trivial as a result of the interdependence of the governing parameters. Substantial research has emerged to increase the figure of merit $Z T$ of thermoelectric materials [2-4], often focusing on the minimization of the thermal conductivity of semiconducting materials while preserving good electronic conduction properties [5,6].

Mixed crystals based on Si and Ge have been studied for their thermoelectric properties starting more than half a century ago [7-9], and they have been of great interest since then [10-13]. The potential of $\mathrm{Si}_{1-x} \mathrm{Ge}_{x}$ alloys for thermoelectric conversion lies in the fact that the thermal conductivity is drastically reduced in the alloy with respect to the pure parent crystals already at small impurity concentration $x$ (or $1-x$, respectively) $[7,8,14-17]$ while maintaining high performance in electronic transport. Additional reduction of the thermal

\footnotetext{
*konstanze.hahn@dsf.unica.it
}

conductivity can be achieved by nanostructuration, hence suppressing the propagation of long-wavelength phonons. Extensive studies have discussed thermoelectric properties in $\mathrm{SiGe}$ superlattices, nanowires, nanotubes, or porous $\mathrm{SiGe}$ materials [18-24]. Recently, the great interest in nanostructured materials has diverted attention from bulk materials, while computational methods have continued to evolve.

Several important experimental studies of bulk SiGe were performed in the second half of the 20th century. They investigated the effect of composition, carrier concentration, and temperature on thermoelectric material properties $[9,25]$. The most elaborate study, by Dismukes et al., covered several Si-rich compositions and carrier concentrations from $2.2 \times 10^{18}$ to $3 \times 10^{20} \mathrm{~cm}^{-3}$ of $n$ - and $p$-type doping [9]. Thermal resistivity, the Seebeck coefficient, electrical resistivity, and Hall mobility were measured. The samples included p-type $\mathrm{Si}_{0.7} \mathrm{Ge}_{0.3}$ and $\mathrm{Si}_{0.85} \mathrm{Ge}_{0.15}$ doped with boron. Carrier concentrations from $2.1 \times 10^{19}$ to $3.5 \times 10^{20} \mathrm{~cm}^{-3}$ were examined for $\mathrm{Si}_{0.7} \mathrm{Ge}_{0.3}$. For $\mathrm{Si}_{0.85} \mathrm{Ge}_{0.15}$ only heavily doped samples were generated with $2.1 \times 10^{20}$ and $3 \times 10^{20} \mathrm{~cm}^{-3}$ doping concentration. For $n$-type materials, $\mathrm{Si}_{0.7} \mathrm{Ge}_{0.3}$ samples were produced with intermediate arsenic doping resulting in carrier concentrations $2.2 \times 10^{18}$ and $2.3 \times 10^{18} \mathrm{~cm}^{-3}$ and heavy doping with phosphorus resulting in carrier concentrations $6.7 \times 10^{19}$ and $1.5 \times 10^{20} \mathrm{~cm}^{-3}$. In addition, two other $n$-type samples were doped with phosphorus, resulting in $\mathrm{Si}_{0.8} \mathrm{Ge}_{0.2}$ with a carrier concentration of $1.4 \times 10^{20} \mathrm{~cm}^{-3}$ and $\mathrm{Si}_{0.85} \mathrm{Ge}_{0.15}$ with a carrier concentration of $2.7 \times 10^{20}$ $\mathrm{cm}^{-3}$. The pure parent materials $\mathrm{Si}$ and Ge were not investigated in this study. The compositions of the studied SiGe alloys all differed slightly from one another, and all samples had different carrier concentrations. For some compositions, only one or two carrier concentrations have been studied. This demonstrates the experimental limitations of conducting a systematic study with the three varying parameters: alloy composition, carrier concentration, and temperature.

Based on these experimental data, empirical models have been developed with the intention of covering a wider and more systematic range of parameters $[10,26]$. Slack and 
Hussein [10] described separate empirical expressions for the carrier mobility and electrical conductivity, the Seebeck coefficient, and thermal conductivity as a function of temperature and carrier concentration with adjustable parameters optimized to match available experimental data. These models were proposed for both $n$ - and $p$-type materials in order to estimate the figure of merit of a thermoelectric generator couple (connected $n$ - and $p$-type unit) focusing only on $\mathrm{Si}_{0.7} \mathrm{Ge}_{0.3}$. On the other hand, a model based on the Boltzmann transport equation (BTE) was presented by Vining [26], where some material-specific parameters were used together with adjustable parameters that were fitted to experimental data. As stated in the study, the proposed model is valid only for heavily doped systems. It was optimized using the data of Dismukes et al. [9] focusing only on Si-rich $n$-type SiGe alloys.

A similar approach was applied by Minnich et al. [18] for the simulation of thermoelectric properties in $\mathrm{Si}_{0.7} \mathrm{Ge}_{0.3}$ bulk and $\mathrm{Si}_{0.8} \mathrm{Ge}_{0.2}$ nanocomposites. In their study, additional data of the electronic conductivity made it possible to estimate the change of carrier concentration with temperature, which was not used in the previous study by Vining [26].

Because of their very nature, empirical studies such as the ones just described rely on selected experimental data: therefore, they can hardly be used as predictive computational studies. In this respect, first-principles calculations based on density functional theory (DFT) have a great potential to be used for systematic studies of abstract properties such as the figure of merit $Z T$ in thermoelectric materials. Given the electronic structure, the Boltzmann transport equation can be solved to yield the electronic transport coefficients. This approach has been applied successfully for the study of $\mathrm{Si}$ and $\mathrm{SiGe}$ nanowires [27,28], in thermoelectric $\mathrm{SrTiO}_{3}$ and $\mathrm{SrRuO}_{3}$ [29,30], and in thermomagnetic MnGeCo [31], to name just a few. The evaluation of the Boltzmann transport equation has been implemented recently in the BOLTZTRAP toolbox [32], allowing postprocessing of the results from DFT calculations.

Shi et al. [27] used DFT calculations and the Boltzmann transport equation to determine the electronic transport coefficients in Si nanowires. At a fixed cross section of $2.3 \mathrm{~nm}^{2}$, $Z T$ had a maximum for a carrier concentration at $10^{19} \mathrm{~cm}^{-3}$. The calculations were only carried out at room temperature, excluding analysis of the temperature effect on the thermoelectric figure of merit $Z T$. The thermal conductivity in this study was taken from previously conducted nonequilibrium molecular-dynamics simulations [33]. In another study using the same method, the authors investigated the figure of merit $Z T$ in SiGe nanowires at room temperature for a fixed cross section [34]. The effect of alloy composition and carrier concentration on $Z T$ was analyzed showing a maximum with respect to $Z T$ of a corresponding Si nanowire at around $0.5 \mathrm{Ge}$ content and a carrier concentration of $2 \times 10^{20} \mathrm{~cm}^{-3}$. Similar to their previous study on Si nanowires, the thermal conductivities were taken from available studies based on molecular dynamics [35].

Very recently, a detailed study was presented by MurphyArmando [13] describing the electronic transport coefficients from $a b$ initio calculations analyzing the effect of carrier concentration and temperature in $n$-type $\mathrm{Si}_{0.7} \mathrm{Ge}_{0.3}$ and the effect of $\mathrm{Si}_{1-x} \mathrm{Ge}_{x}$ alloy composition $x$ at a given temper- ature $(1300 \mathrm{~K})$ and carrier concentration $\left(10^{20} \mathrm{~cm}^{-3}\right)$. The study also investigated the figure of merit in the strained $\mathrm{Si}_{1-x} \mathrm{Ge}_{x}$ alloy. An empirical expression for the lattice thermal conductivity was fitted instead to the experimental data of Dismukes et al. [9].

In the studies described above, the lattice thermal conductivity has either been taken from experimental data, described by empirical models fitted to experiments or calculated using molecular-dynamics (MD) simulations [36,37], which are also based on empirical force fields. In this latter case, equilibrium [38-40], nonequilibrium [39-42], and approachto-equilibrium MD simulations $[17,43,44]$ have been used. A consistent basis for the study of thermoelectrics is thus not provided.

A promising alternative approach to classical simulations is the application of density functional perturbation theory (DFPT) for the determination of second- and third-order interatomic force constants applied to the phonon Boltzmann transport equation for three-phonon scattering terms. This fully first-principles approach has been successfully applied for bulk SiGe [16] and implemented within the single-mode relaxation time approximation and a variational approach in the QUANTUM ESPRESSO program package $[45,46]$. A similar computational setup was applied recently to the calculation of the thermoelectric figure of merit in $\mathrm{Si}_{0.75} \mathrm{Ge}_{0.25}$ [12]. In the latter study, the nearly-free-electron model was used to determine electronic transport, and, in addition to anharmonic scattering events from the third-order interatomic force constants, boundary scattering and harmonic scattering from mass disorder, vacancies, and other impurities was considered for the calculation of the lattice thermal conductivity. The figure of merit as a function of temperature was calculated for the $n$-type $\mathrm{Si}_{0.75} \mathrm{Ge}_{0.25}$ sample with a carrier concentration of $9.4 \times 10^{19} \mathrm{~cm}^{-3}$.

In summary, a large number of experimental and theoretical studies have been published on the thermoelectric properties in SiGe-based materials, each one, however, limited either by the factors (temperature, carrier concentration, alloy composition) considered in the evaluation of $Z T$ or by the consistency of the methods applied separately for electronic transport and lattice thermal conductivity.

Motivated by this state of affairs, we provide a systematic and consistent study based on first-principles calculations of the intrinsic thermoelectric properties in ideal (i.e., defect-free) $n$ - and $p$-type bulk SiGe compositional alloys. Electronic and thermal transport coefficients have been evaluated using the electronic and phonon Boltzmann transport equation, respectively. The Seebeck coefficient and electrical and thermal conductivity have been calculated focusing on the effect of alloy composition, carrier concentration, and temperature, eventually leading to the determination of the thermoelectric figure of merit as a function of the three parameters.

\section{THEORETICAL FRAMEWORK}

We present a comprehensive study of electronic and thermal transport coefficients leading to a consistent and systematic description of the thermoelectric figure of merit $Z T$ in bulk SiGe. Most of the calculations are performed relying on 
first-principles consistence. For computational cost-efficiency, the only ingredient obtained from empirical expressions is the electron lifetime as discussed thoroughly in section I, Theoretical Framework, of the supplemental material [47] together with all other implementation details.

Both electronic and thermal transport coefficients are calculated based on the electronic and phonon properties obtained from $\mathrm{DF}(\mathrm{P}) \mathrm{T}$ calculations. In particular, DFT calculations have been carried out using the QUANTUM ESPRESSO suite of programs [48] for ground-state calculations of several SiGe alloys. The local density approximation has been applied to model the exchange and correlation interaction [49], together with norm-conserving Trouiller-Martins type pseudopotentials [50]. Monkhorst-Pack sampling has been applied for the k-point grid [51].

To account for varying $\mathrm{Si}$ and $\mathrm{Ge}$ concentrations, the virtual crystal approximation (VCA) has been applied as implemented in the QUANTUM ESPRESSO program package allowing the use of a primitive unit cell containing two atoms for any concentration in the alloy. This implies that the core of the atoms in the virtual crystal is described by an average pseudopotential generated from the pseudopotentials of the elemental components weighted by their concentration in the alloy (see the supplemental material [47] for more details). A pseudopotential of the virtual crystal for the $\mathrm{Si}_{1-x} \mathrm{Ge}_{x}$ alloy was generated for each $x$ prior to the DFT calculations. The lattice structure of the virtual crystal has translational invariance and periodic properties (space group $F d \overline{3} m$ ). In the VCA, a solid solution composed of different atom types is approximated with an elemental crystal made of just one sole virtual element (see Fig. S1 of the supplemental material [47]) whose scattering matrix is a combination of the matrices from the component elements [52]. VCA has proved to be both efficient and reliable for the calculation of electronic and structural properties of alloyed structures [16,53,54]. It describes an ideal, boundary-free, and defect-free crystal considering the average electronic properties of the composing atoms.

Based on the electronic structure obtained from the DFT calculations and the VCA approach for different SiGe alloys, the electronic Boltzmann transport equation has been solved within the relaxation time approximation (RTA) using the BoltzTrAP toolbox [32]. Use of the RTA is justified in systems close to equilibrium, which is the case in solids and is equally applicable for phonon transport. The relaxation time has been derived from an empirical expression of the temperature- and carrier-dependent alloy mobility $[10,55,56]$ as already successfully applied to determine the relaxation time in SiGe-based thermoelectric materials [34] in intermediate-to-high temperatures $(T \geqslant 100 \mathrm{~K})$ where Coulomb scattering can be neglected. Details about the theory behind it are described in the supplemental material [47].

Electronic transport coefficients are highly sensitive to the magnitude of the band gap. In standard DFT calculations, as performed here, the band gap of a semiconductor is underestimated as a result of the self-interaction error. For the calculation of the electronic transport coefficients, this error has been corrected by suitably shifting the conduction bands to result in a $\mathrm{Si}_{1-x} \mathrm{Ge}_{x}$ band gap given by a previously proposed relation [57].
The phonon transport processes have been evaluated using the D3Q plug-in of the QUANTUM ESPRESSO program package, which provides the possibility to estimate third-order interatomic force constants allowing for the determination of three phonon scattering processes and eventually the calculation of the phonon relaxation time and thermal conductivity as described below. In this approach, the thermal conductivity is determined using DFPT within the so-called " $2 n+1$ " approach [58,59] and accordingly calculating the anharmonic scattering coefficients for three arbitrary wave vectors [45,46,60-62]. Compositional defects in the alloys have been described through a two-step procedure: at first, the actual stoichiometry is used to define the virtual atom, then mass disorder is accounted for by adding a harmonic scattering term that properly takes into consideration the mass difference between $\mathrm{Si}$ and $\mathrm{Ge}$ and their respective concentration. The harmonic scattering term resulting from mass disorder is more extensively described in the supplemental material [47]. Lattice defects (namely, imperfections with respect to an ideal diamond lattice decorated by VCA atoms such as, e.g., vacancies, self-interstitials, grain boundaries, amorphous pockets, or impurities), other than intended doping ones, are not considered in this study. The obtained intrinsic lattice thermal conductivity can therefore be considered an upper limit with respect to experimental results obtained from real materials.

The phonon BTE has been applied within the single mode relaxation time approximation (SMA) [63-65]. Here, temperatures from 100 to $1200 \mathrm{~K}$ have been considered, focusing, however, on the technologically relevant temperatures $\geqslant 300 \mathrm{~K}$. At such temperatures, umklapp processes dominate the phonon scattering justifying the use of the SMA [66]. The variational approach described by Fugallo et al. [46] has been applied to selected $\mathrm{Si}, \mathrm{Ge}$, and $\mathrm{SiGe}$ systems showing no substantial difference in the resulting thermal conductivity with respect to SMA in the temperature range of interest.

Based on this framework, transport coefficients and the corresponding figure of merit $Z T$ have been systematically studied for carrier concentrations from $10^{15}$ to $10^{20}$ $\mathrm{cm}^{-3}$, temperatures from 100 to $1200 \mathrm{~K}$, and seven different $\mathrm{Si}_{1-x} \mathrm{Ge}_{x}$ alloys $(x=0,0.1,0.25,0.5,0.75,0.9,1)$. Both $n$ - and $p$-type materials have been calculated. Results of $p$-type $\mathrm{SiGe}$ alloys can be found in the supplemental material [47].

\section{ELECTRONIC TRANSPORT}

\section{A. Seebeck coefficient \\ 1. Carrier dependence}

With increasing chemical potential (approaching the minimum of the conduction band), the absolute value of the Seebeck coefficient in $n$-type semiconductors decreases: this occurs by increasing the level of doping (concentration of carriers). The results obtained here for $\mathrm{Si}_{0.75} \mathrm{Ge}_{0.25}$ are in very good agreement with previous experimental and theoretical results at $300 \mathrm{~K}$ (Fig. S2). An exponential decrease of the absolute value of the Seebeck coefficient is expected with increasing carrier concentrations for chemical potentials below the conduction band and above the valence band in $n$ - and 


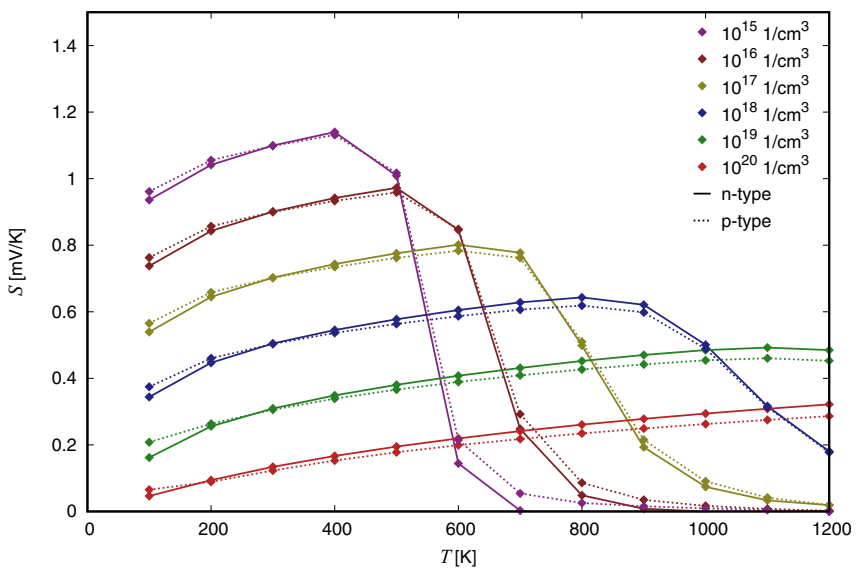

FIG. 1. Absolute value of the Seebeck coefficient at $300 \mathrm{~K}$ as a function of temperature for $n$-type (solid lines) and $p$-type (dotted lines) $\mathrm{Si}_{0.75} \mathrm{Ge}_{0.25}$ at various carrier concentrations.

$p$-type materials, respectively. This behavior is verified for the Seebeck coefficient calculated here for all SiGe alloys, both $n$ - and $p$-type (some are represented in Fig. S3).

A notable decrease of the Seebeck coefficient is observed for $\mathrm{Si}_{0.1} \mathrm{Ge}_{0.9}$ and $\mathrm{Ge}$, in particular in the $n$-type samples (Fig. S4). This can be explained by the switch of the conduction-band minimum positioned at $\Delta$ for $x<0.85$ to $L$ for $x>0.85$.

\section{Temperature dependence}

Figure 1 shows the absolute value of the Seebeck coefficient for various carrier concentrations in $n$-type and p-type $\mathrm{Si}_{0.75} \mathrm{Ge}_{0.25}$ as a function of temperature (note that $S$ is negative in $n$-type materials). In all materials, the Seebeck coefficient is symmetric, giving a similar temperature dependence for both $n$ - and $p$-type doping. At the lowest carrier concentration calculated here $\left(N=10^{15} \mathrm{~cm}^{-3}\right)$, a steep decrease in the Seebeck is observed at $600 \mathrm{~K}$. This is attributed to a significant decrease of the chemical potential in the extrinsic regime converging eventually to the Fermi energy of the intrinsic material. With increasing doping concentration, the drop of the Seebeck coefficient shifts to higher temperatures, consistent with the extrinsic regime extending to higher temperatures at higher doping levels. In heavily doped materials $\left(N=10^{19}\right.$ and $\left.10^{20} \mathrm{~cm}^{-3}\right)$, the extrinsic regime extends beyond the temperatures calculated here $(T \leqslant 1200 \mathrm{~K})$.

The chemical potential in the extrinsic regime is $\mu_{c}=E_{C, V} \pm k_{B} T \ln \frac{N_{C}}{N}$, where $E_{C, V}$ is the conduction-band minimum and the valence-band maximum, respectively, and $N_{C, V}$ are the effective carrier densities in the respective bands indicating a steeper gradient with decreasing doping concentration $N$. This behavior is confirmed by the results presented in Fig. 1.

The Seebeck coefficient for $n$-type SiGe has been calculated as a function of the temperature for various $\mathrm{SiGe}$ alloys at a carrier concentration of $N=10^{18} \mathrm{~cm}^{-3}$ (Fig. S5). The maximum found around $800 \mathrm{~K}$ in $\mathrm{Si}_{0.75} \mathrm{Ge}_{0.25}$ (see Fig. 1) shifts to lower temperatures with increasing $x$ (Ge content), and its absolute value decreases slightly. The same trend is observed for $p$-type samples (Fig. S6).
Interestingly, the Seebeck coefficient in $\mathrm{Ge}$ and $\mathrm{Si}_{0.1} \mathrm{Ge}_{0.9}$ is smaller than in SiGe alloys with less Ge content at low temperatures, but it surpasses the other alloys at higher temperatures $(T>1000 \mathrm{~K})$. This can be explained by the differences in the effective mass of electrons, which changes with the position of the minimum in the conduction band as mentioned before.

\section{Effect of the alloy composition}

The effect of the Ge content $x$ in the $\mathrm{Si}_{1-x} \mathrm{Ge}_{x}$ alloy on the Seebeck coefficient in $n$-type and $p$-type materials has been specifically analyzed for a carrier concentration of $N=10^{18} \mathrm{~cm}^{-3}$ (Fig. S7). At room temperature $(300 \mathrm{~K})$, the Seebeck coefficient is nearly constant for all $\mathrm{Si}_{1-x} \mathrm{Ge}_{x}$, in agreement with Ref. [13]. A slight increase in $n$-type materials is observed close to $x=0.85$, which can be explained by an increase of the density of states at the band crossover (from $\Delta$ to $L$ ).

At a temperature of $1000 \mathrm{~K}$, the Seebeck coefficient shows a steady decrease with increasing $x$ (Ge content), similar to previous results [13]. In $p$-type materials, the decrease at higher $x$ is more pronounced with respect to the $n$-type materials. As discussed before, the reduced decrease in $n$-type materials is most likely a result of the band crossover close to $x=0.85$. It is expected that at higher temperatures, even an increase of the Seebeck coefficient with increasing Ge content at $x>0.85$ can be observed (Fig. S5).

A summary of the behavior of the Seebeck coefficient affected by carrier concentration, temperature, and alloy composition is shown in Fig. 2 for $n$-type materials (see Fig. S8 for $p$-type). At constant carrier concentration (first column of panels), $S$ is nearly independent of the alloy composition. The highest values around $1.2 \mathrm{mV} / \mathrm{K}$ are observed in $\mathrm{Si}$ rich materials at low carrier concentration and intermediate temperatures (200-500 K).

\section{B. Electrical conductivity}

\section{Carrier dependence}

An increase of the carrier concentration results in an increase of the electrical conductivity, described by a linear relation ( $\sigma \sim N$, Fig. 3). The electrical conductivity calculated here using the BTE model confirms the proportionality of the two (note the semilogarithmic scale in Fig. 3). The obtained results for $\mathrm{Si}_{0.75} \mathrm{Ge}_{0.25}$ are in excellent agreement with previous experimental [9] and theoretical [67] data for $\mathrm{Si}_{0.7} \mathrm{Ge}_{0.3}$ samples.

This behavior is confirmed for all SiGe alloys. The electrical conductivity of $n$-type materials (Fig. S9) in general is higher with respect to their $p$-type counterparts (Fig. S10). For all calculated $n$-doping concentrations, $\sigma$ is highest in Ge.

\section{Temperature dependence}

The temperature dependence of the electrical conductivity has been analyzed in detail at an intermediate carrier concentration of $N=10^{18} \mathrm{~cm}^{-3}$ (Fig. S11 n-type, Fig. S12 $p$-type). We provide evidence that a temperature increase leads to a decrease in the electrical conductivity up to around $800 \mathrm{~K}$. Further heating results in an increase of the 

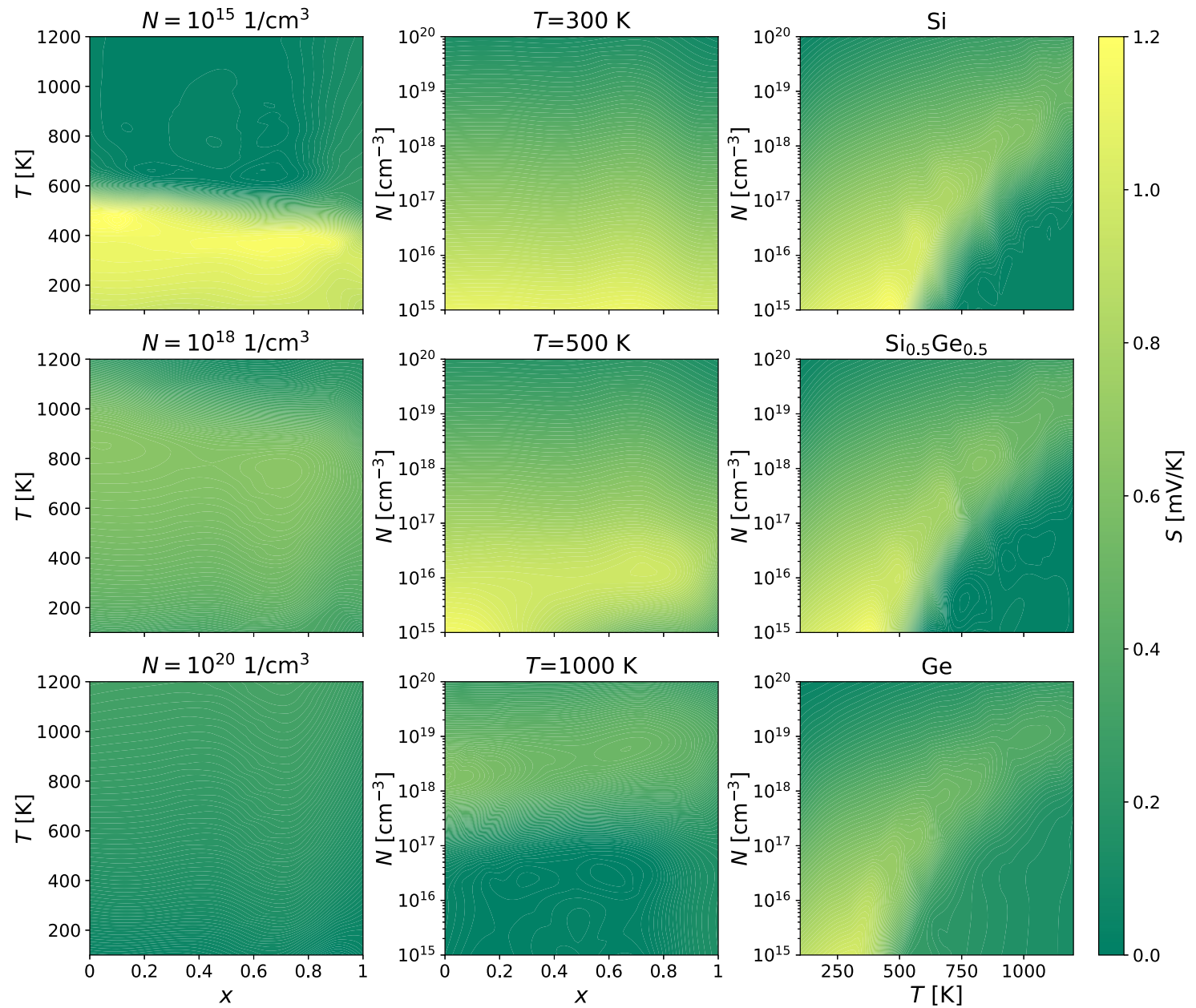

FIG. 2. Absolute value of the Seebeck coefficient of $n$-type $\mathrm{Si}_{1-x} \mathrm{Ge}_{x}$ alloys with varying carrier concentration $N$, Ge content $x$, and temperature $T$.

electrical conductivity. The decrease at lower temperatures can be attributed to increased phonon scattering and thus a reduction of the mobility. At higher temperatures, the

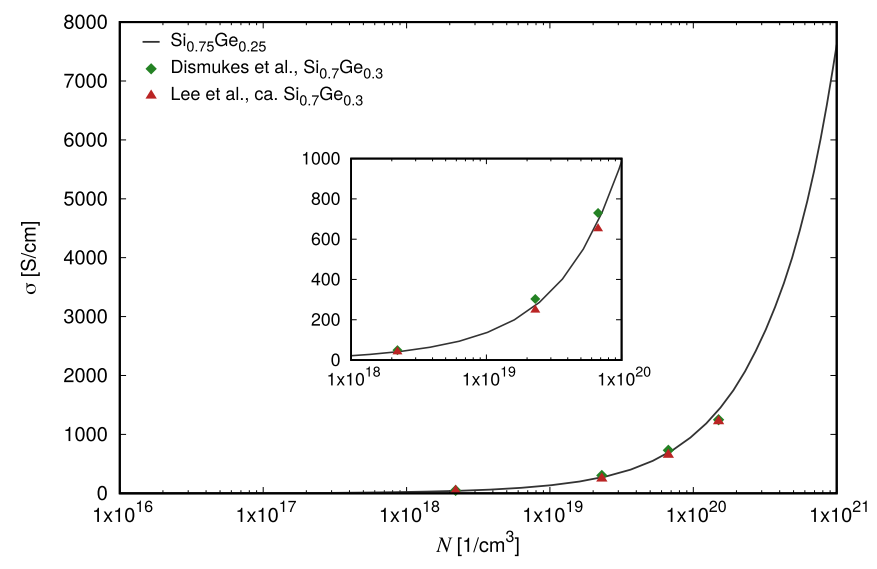

FIG. 3. Electrical conductivity at $300 \mathrm{~K}$ as a function of carrier concentration in $\mathrm{Si}_{0.75} \mathrm{Ge}_{0.25}$ in comparison with previous experimental [9] and theoretical [67] studies. The inset shows a magnified view of the range $10^{18} \leqslant N \leqslant 10^{20} \mathrm{~cm}^{-3}$ for better visualization of the results. conductivity is dominated by the total number of carriers in the material, which increases with increasing temperature as a result of increasing intrinsic carriers. The minimum in electrical conductivity at around $800 \mathrm{~K}$ is most pronounced in pure Ge.

\section{Effect of the alloy composition}

To properly describe carrier transport in semiconductors, various scattering mechanisms have to be considered. A detailed analysis of several intra- and intervalley scattering mechanisms in SiGe alloys has been previously presented [68]. At room temperature ( $300 \mathrm{~K})$, the mobility of $n$-type materials was shown to be reduced in the SiGe alloys with respect to the pure counterparts $\mathrm{Si}$ and $\mathrm{Ge}$ in agreement with experimental results $[69,70]$. In particular, a remarkable decrease of carrier mobility was observed already at low Si content with respect to pure Ge. Despite the complexity of scattering mechanisms affecting the carrier mobility in $\mathrm{SiGe}$, the simplified model used here is capable of describing well the change of carrier mobilities in $\mathrm{Si}_{1-x} \mathrm{Ge}_{x}$ with varying $x$ in particular at intermediate to high doping levels $\left(N>10^{19} \mathrm{~cm}^{-3}\right.$, Fig. S13).

The reduced mobility in SiGe alloys leads to a similar behavior in the electrical conductivity. Trends of the electrical 

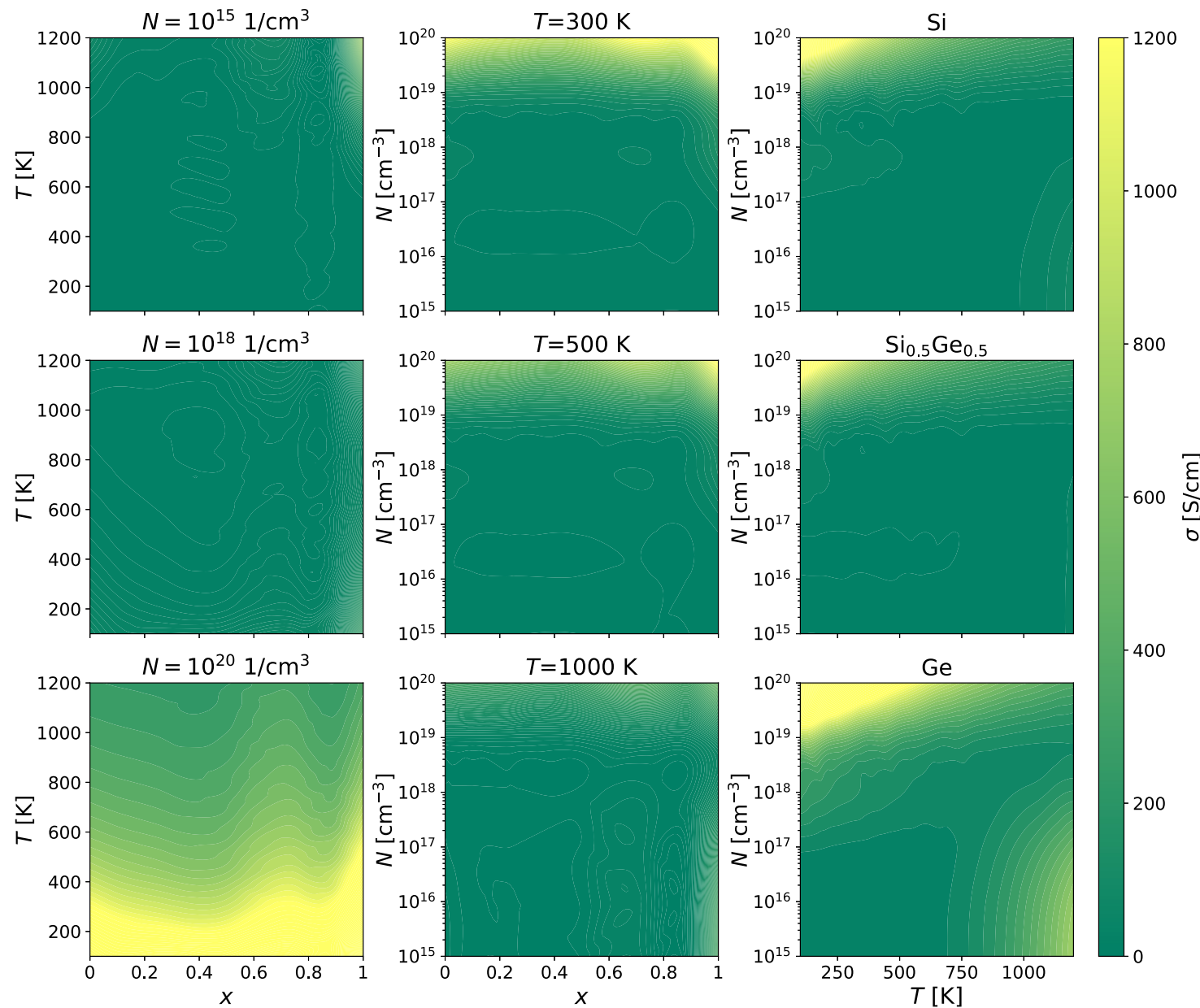

FIG. 4. Electrical conductivity of $n$-type $\mathrm{Si}_{1-x} \mathrm{Ge}_{x}$ alloys with varying carrier concentration $N$, Ge content $x$, and temperature $T$.

conductivity in SiGe alloys calculated here at a carrier concentration of $N=10^{18} \mathrm{~cm}^{-3}$ at 300 and $1000 \mathrm{~K}$ (Fig. S14) are similar to results reported previously for $n$-type SiGe alloys at $1200 \mathrm{~K}$ [13].

In summary, $\sigma$ is mostly controlled by the carrier concentration giving insignificant values in intermediate and lightly doped materials $\left(N \leqslant 10^{18} \mathrm{~cm}^{-3}\right)$ as shown in Fig. 4. The highest values are observed in Ge-rich alloys at low temperatures. A similar behavior is observed in $p$-type materials (Fig. S15).

\section{Power factor}

The power factor given by $\sigma S^{2}$ describes the electronic features entering in the thermoelectric figure of merit $Z T$. It is independent of the thermal transport properties and offers the possibility of comparing and verifying the electronic contribution to the thermoelectric properties. We have calculated it specifically for $\mathrm{Si}_{0.75} \mathrm{Ge}_{0.25}$ at 300 and $1000 \mathrm{~K}$ as a function of the carrier concentration (Fig. $5 n$-type, Fig. S16 $p$-type), and we obtained very good agreement with previous experimental results [9]. At room temperature, it shows a maximum between $10^{19}$ and $10^{20} \mathrm{~cm}^{-3}$. The maximum is higher and shifted to higher carrier concentrations $\left(\sim 10^{21}\right.$ $\mathrm{cm}^{-3}$ ) when increasing the temperature up to $1000 \mathrm{~K}$. Anal- ysis of the temperature dependence of the power factor in $p$-type $\mathrm{SiGe}$ shows as well very good agreement with previous experimental results [71] (Fig. S17). This result stands for the overall reliability, transferability, and accuracy of the present theoretical and computational setup.

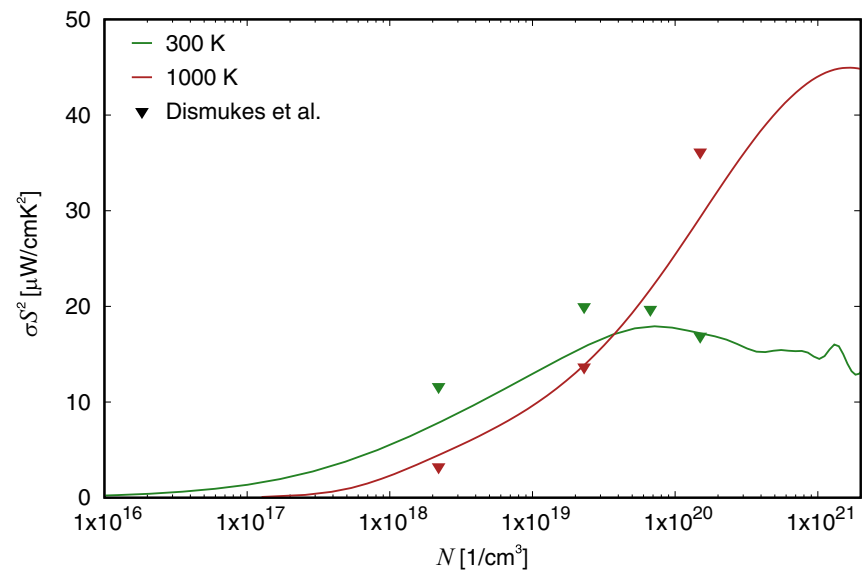

FIG. 5. Power factor $\sigma S^{2}$ as a function of carrier concentration $N$ in $n$-type $\mathrm{Si}_{0.75} \mathrm{Ge}_{0.25}$ at $300 \mathrm{~K}$ (green) and $1000 \mathrm{~K}$ (red) in comparison to experimental data for samples close to $\mathrm{Si}_{0.7} \mathrm{Ge}_{0.3}$ by Dismukes et al. [9] (triangles). 


\section{THERMAL TRANSPORT}

\section{A. Effect of mass disorder}

As described in Sec. II (Theoretical Framework), the lattice thermal conductivity has been calculated using DFPT to determine the second (harmonic) and third (anharmonic) order interatomic force constants, which are then used to solve the phonon BTE. The DFPT calculations are carried out in the framework of the virtual crystal approach. Assigning an average mass to virtual crystal atoms, however, drastically reduces phonon scattering events resulting from mass disorder, and as a result yields an overestimated thermal conductivity. Mass disorder has been added in the calculations as an additional harmonic scattering term as described above and in the supplemental material [47]. This approach has been used successfully in SiGe alloys [16] and other materials such as lead-based alloys [72] and $\mathrm{Mg}_{2} \mathrm{Si}_{x} \mathrm{Sn}_{1-x}$ [73] showing good agreement with experiments. It has been demonstrated that in alloys composed of two group IV elements, perturbation from mass disorder is sufficient to accurately describe the thermal conductivity, whereas in alloys composed of III-V or II-VI group elements, such as $\operatorname{In}_{1-x} \mathrm{Ga}_{x}$ As, additional force constant disorder needs to be taken into account [74]. A very recent detailed study compared several advanced methods including the $T$-matrix approach and the supercell unfolding method, showing good agreement in phonon lifetimes for low-energy phonons [75]. This confirms that all methods, including the approach used here, are reliable in SiGe alloys where 90\% of the lattice thermal conductivity can be attributed to lowenergy phonons [75].

Our calculations confirmed that mass disorder scattering drastically reduces the thermal conductivity in $\mathrm{Si}_{1-x} \mathrm{Ge}_{x}$ alloy. Negligence of the latter resulted in a more than sevenfold overestimation of the thermal conductivity in certain samples.

Increased scattering from mass disorder is expected with increased doping concentration and accordingly has been included in the calculations of the lattice thermal conductivity for heavily doped systems. In the SiGe alloys studied here $(x=0.1,0.25,0.5,0.75$, and 0.9$)$, even the highest carrier concentration considered $\left(N=10^{20} \mathrm{~cm}^{-3}\right)$ resulted in a variation of the lattice thermal conductivity by only $1 \%$. In the alloys it has thus been neglected. In pure $\mathrm{Si}$ and Ge, however, the doping concentration needs to be considered in the calculation of the lattice thermal conductivity. Several doping concentrations $N$ have been calculated for doping with arsenic (As) in Si (Fig. S19). A remarkable decrease in the thermal conductivity is observed for doping concentrations of $10^{19} \mathrm{~cm}^{-3}$ and higher.

In heavily doped $\mathrm{Ge}\left(4.6 \times 10^{20} \mathrm{~cm}^{-3}\right)$, As-doping results in an insignificant reduction of the thermal conductivity by $0.6 \%$. This confirms the impact of mass difference between host and dopant, which is very low between Ge and As, having atomic masses of 72.64 and 74.92 a.u., respectively. However, the thermal conductivity in $\mathrm{Ge}$ is notably reduced when doped with phosphorus $(\mathrm{P})$. At a $\mathrm{P}$ concentration of $4.6 \times 10^{20} \mathrm{~cm}^{-3}$, the thermal conductivity is reduced by $52 \%$ with respect to undoped Ge (Fig. S19).

Based on these results, the lattice thermal conductivity for the calculation of the figure of merit has been considered unaltered with respect to the undoped material for all $\mathrm{SiGe}$ alloys and for pure $\mathrm{Si}$ and $\mathrm{Ge}$ up to a doping concentration of $10^{17} \mathrm{~cm}^{-3}$. At doping concentrations of $10^{18} \mathrm{~cm}^{-3}$ and higher, a relative reduction of the thermal conductivity has been assumed based on the results for As and P doping in $\mathrm{Si}$ and Ge, respectively.

Phonon-electron interactions have not been considered in the calculation of the lattice thermal conductivity. Such interactions have been shown to be negligible for charge carrier concentrations below $10^{18} \mathrm{~cm}^{-3}$. On the other hand, it has been reported that the calculated lattice thermal conductivity for higher carrier concentration is in fact affected by phonon-electron scattering [76]. The results presented here can therefore be regarded as an upper limit of the lattice thermal conductivity.

\section{B. Temperature dependence}

The present calculations have been performed in the temperature range from 100 to $1200 \mathrm{~K}$. At low temperatures, the lattice thermal conductivity increases with increasing temperature as a result of higher phonon group velocities. Heat transport in this temperature range is dominated by normal processes. The maximum of thermal conductivity in $\mathrm{Si}$ and $\mathrm{Ge}$ is found around 25 and $10 \mathrm{~K}$, respectively [77]. In the temperature range calculated here $(T \geqslant 100 \mathrm{~K})$, lattice thermal conductivity is dominated by umklapp scattering, which increases with increasing temperature resulting in a decreasing thermal conductivity. A drastic decrease is observed in particular between 100 and $300 \mathrm{~K}$. Results of $\mathrm{Si}$ and $\mathrm{Ge}$ are in excellent agreement with experimental results (Fig. S20) [77-79].

\section{Effect of the alloy composition}

The effect of the SiGe alloy composition on its lattice thermal conductivity has been studied extensively both experimentally [8,14] and theoretically [16,17]. Already at small $\mathrm{Si}$ or $\mathrm{Ge}$ content, i.e., close to pure $\mathrm{Ge}$ and $\mathrm{Si}$, respectively, the thermal conductivity is notably reduced with respect to the pure materials. This remarkable decrease is mainly caused by mass disorder, confirmed by the calculations performed here. Without considering the effect of mass disorder, the thermal conductivity would decrease linearly with increasing Ge content.

The lattice thermal conductivity calculated here is in excellent agreement with experimental data from Steele and Rosi [80] at room temperature (300 K) (Fig. 6) and below. Several other experimental studies have shown lower values of the thermal conductivity $[9,25,81]$, in some cases less than half of the values calculated here. Reported values for Si-rich alloys $(0.15 \leqslant x \leqslant 0.3)$ at room temperature range from 6 to $19 \mathrm{~W} / \mathrm{mK}[9,80,81]$. The differences in the production method and the resulting amount of impurities and defects in the samples is likely the reason for the discrepancies between experimental studies. This guess is confirmed by a study demonstrating that the thermal conductivity in sintered SiGe alloys can be half that of crystalline $\mathrm{SiGe}$ produced by other methods [25].

This issue has been addressed recently also from a theoretical point of view, focusing on the lowest possible value (the so-called alloy limit) of the thermal conductivity in SiGe 


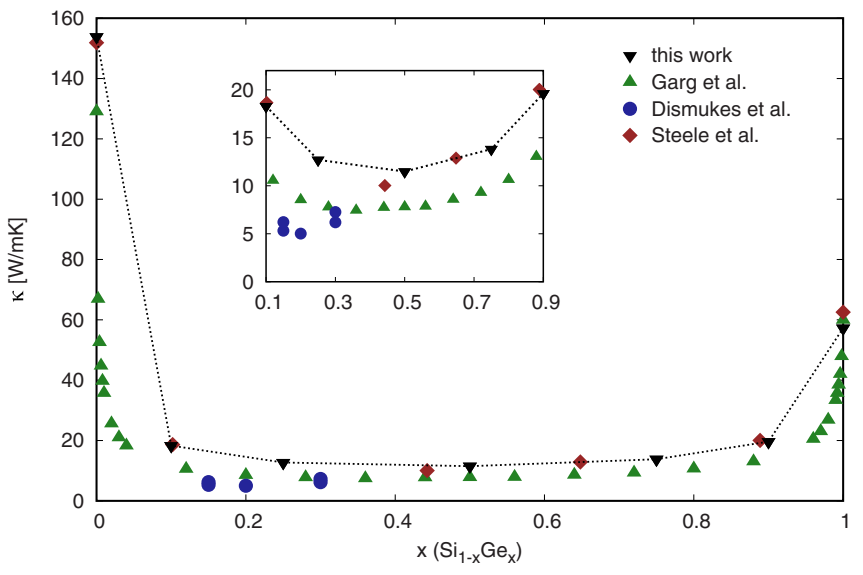

FIG. 6. Thermal conductivity in $\mathrm{Si}_{1-x} \mathrm{Ge}_{x}$ alloys as a function of $x$ at $300 \mathrm{~K}$ in comparison to previous experimental $[9,80]$ and theoretical [16] studies. The inset shows a magnified view of the range $0.1 \leqslant x \leqslant 0.9$ for better visualization of the differences in lattice thermal conductivity between various experimental and theoretical studies.

alloys, which has been examined by MD simulations [82]. The latter study showed a notable decrease in the lattice thermal conductivity when simulation cells were composed of larger units with irregular atom distribution, demonstrating the importance of a sufficiently large unit cell. Furthermore, another study based on MD simulations has shown that the thermal conductivity is drastically reduced when a second phase is formed, introducing additional scattering events at grain boundaries [17]. These theoretical explanations are in line with experimental studies stating that some specimens exhibit small amounts of a second phase and consist of elongated grains [9]. Such structural effects cannot be captured explicitly in first-principles calculations due to limitations of the simulation unit.

Several approaches have been discussed recently to overcome this issue. Scattering effects from vacancies and impurities, for example, have been introduced in a firstprinciples study [12]; however, they rely on a parameter that must be adjusted based on experimental results [83]. More advanced methods applying the $T$-matrix method for point defects have shown that, in particular, vacancies can lead to a drastic decrease in the thermal conductivity $[84,85]$. Our study is intended to give intrinsic values of the lattice thermal conductivity, therefore such effects have been neglected.

Discrepancies with other theoretical studies using the same approach as in this study result from a different q-grid used for the calculation of the thermal conductivity [Eq. (18) in the supplemental material [47]]. In our study, the lattice thermal conductivity has been extrapolated to an infinite grid as explained in the supplemental material [47] (see Fig. S22), while previous studies often used finite values corresponding to q-grids between $10 \times 10 \times 10$ and $20 \times 20 \times 20$ mainly chosen for convenience [16,86]. Using a q-grid of $20 \times 20 \times 20$, our results are in excellent agreement with previous results [16] (Fig. S23), however they are remarkably lower than those obtained by an extrapolation to an infinite q-grid (Fig. 6, inset), which we consider here as the ultimate value.
Finally, we remark that even adopting a similar MD simulation approach, the predicted lattice thermal conductivity of a $\mathrm{Si}_{0.5} \mathrm{Ge}_{0.5}$ homogeneous alloy is reported to vary in a wide range of values, namely $1-7 \mathrm{~W} / \mathrm{mK}[17,40,43,87]$. This points out how the MD predicted value of thermal conductivity depends on many implementation details, such as, for example, the force field, the simulations cell size, and the equilibrium (Green-Kubo) or nonequilibrium (direct method) protocol of the simulation.

The overall thermal conductivity given by the sum of the lattice thermal conductivity and the electronic thermal conductivity is represented in Fig. 7. At room temperature, the electronic contribution to the thermal conductivity plays a minor role. However, it becomes more significant at high temperatures, particularly in combination with heavy doping (Figs. S25 and S26). At $1000 \mathrm{~K}$ and a carrier concentration of $N=10^{20} \mathrm{~cm}^{-3}$, the electronic contribution to the thermal conductivity in $\mathrm{Si}_{0.25} \mathrm{Ge}_{0.75}$ is more than one third in both $n$ - and $p$-type materials.

\section{FIGURE OF MERIT}

\section{A. Carrier dependence}

Based on the transport coefficients discussed in the previous paragraphs, $Z T$ has been calculated for varying parameters. The values for $Z T$ can be regarded as a lower limit since the thermal conductivity calculated here is based on intrinsic properties and represents an upper limit for the latter. Figure 8 shows $Z T$ as a function of the carrier concentration in $\mathrm{Si}_{0.75} \mathrm{Ge}_{0.25}$ at a temperature of 300 and $1000 \mathrm{~K}$. At room temperature, a maximum of $Z T$ is observed between $10^{19}$ and $10^{20} \mathrm{~cm}^{-3}$, corresponding to the maximum of the power factor (Fig. 5), where the maximum in the $p$-type material is at somewhat lower concentrations and in magnitude slightly smaller than in the $n$-type material. In agreement with previous experimental and theoretical studies [9,13,26], $Z T$ is higher at elevated temperatures (here $1000 \mathrm{~K}$ ), and the maximum is found at higher carrier concentrations. At $1000 \mathrm{~K}$, a maximum $Z T$ in $\mathrm{Si}_{0.75} \mathrm{Ge}_{0.25}$ of 0.3 is calculated for an $n$-type carrier concentration of $3.7 \times 10^{20} \mathrm{~cm}^{-3}$. This value is notably lower with respect to previously reported data mainly resulting from the differences in the thermal conductivity as discussed in the previous section and confirmed by the agreement in the power factor (Figs. 5, S16, and S17).

\section{B. Temperature dependence}

In general, $Z T$ in SiGe alloys is higher with respect to the pure $\mathrm{Si}$ and $\mathrm{Ge}$ as a result of the reduced thermal conductivity. However, at $300 \mathrm{~K}$, it is fairly low in all bulk SiGe alloys, making such materials irrelevant for technological applications at room temperature. With increasing temperature, the thermal conductivity decreases in all materials, in particular in Ge-rich systems, leading to a notable increase in $Z T$. At $n$-type doping of $10^{18} \mathrm{~cm}^{-3}$, the highest $Z T$ (0.057) is found for pure $\mathrm{Ge}$ at $800 \mathrm{~K}$, followed by $\mathrm{Si}_{0.25} \mathrm{Ge}_{0.75}$ with a slightly lower maximum of 0.05 at the same temperature (Fig. S28). Increasing $\mathrm{Si}$ content in the $\mathrm{SiGe}$ alloy in general results in a decrease of the $Z T$ peak and its shift to higher temperatures. 

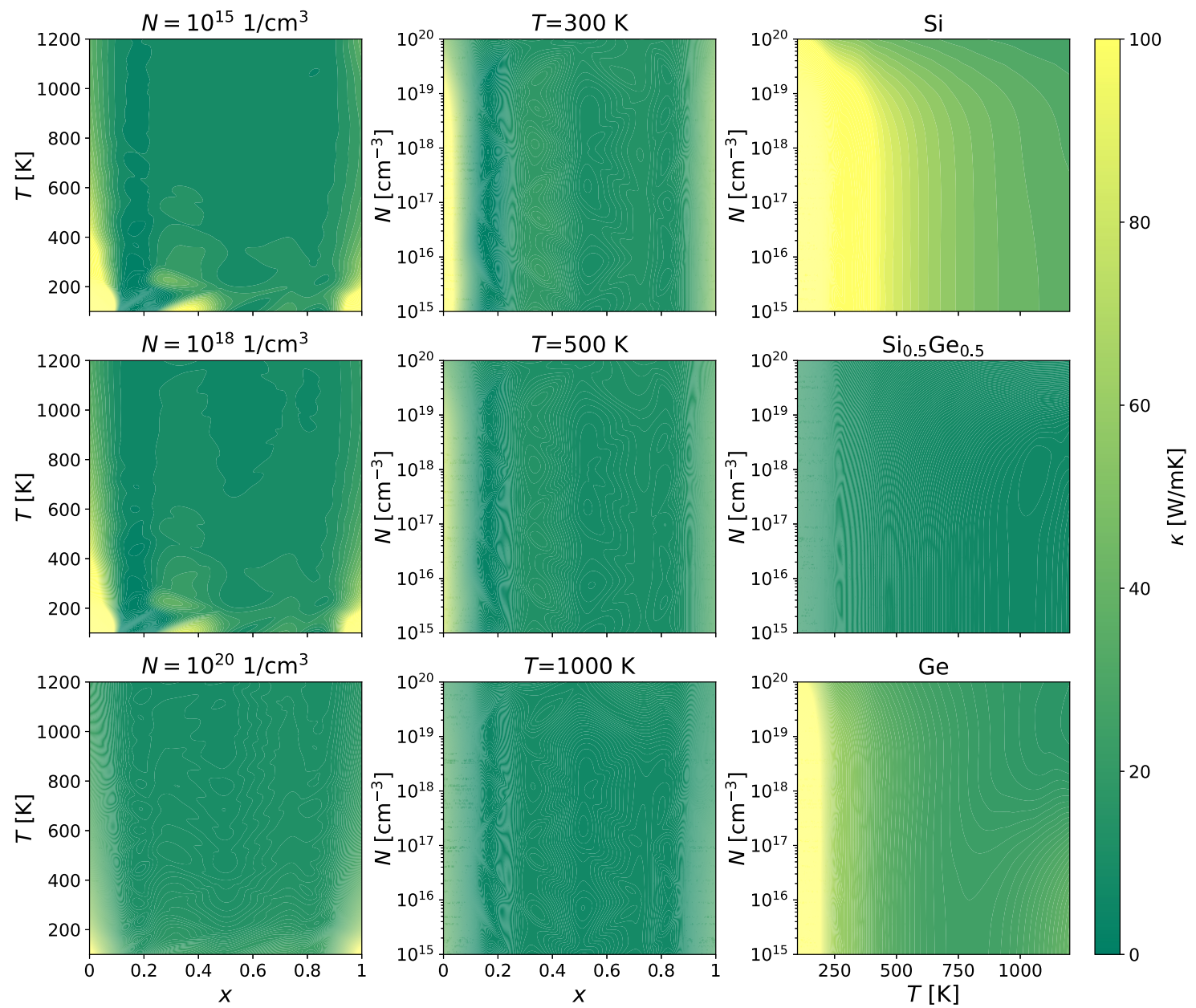

FIG. 7. Overall thermal conductivity $\left(\kappa_{e}+\kappa_{L}\right)$ of $n$-type $\mathrm{Si}_{1-x} \mathrm{Ge}_{x}$ alloys with varying carrier concentration $N$, Ge content $x$, and temperature $T$.

\section{Effect of the alloy composition}

The effect of the alloy composition on $Z T$ has been analyzed in detail for a carrier concentration of $10^{18} \mathrm{~cm}^{-3}$ at 300 and $1000 \mathrm{~K}$ (Fig. S30). At both temperatures, $Z T$ increases

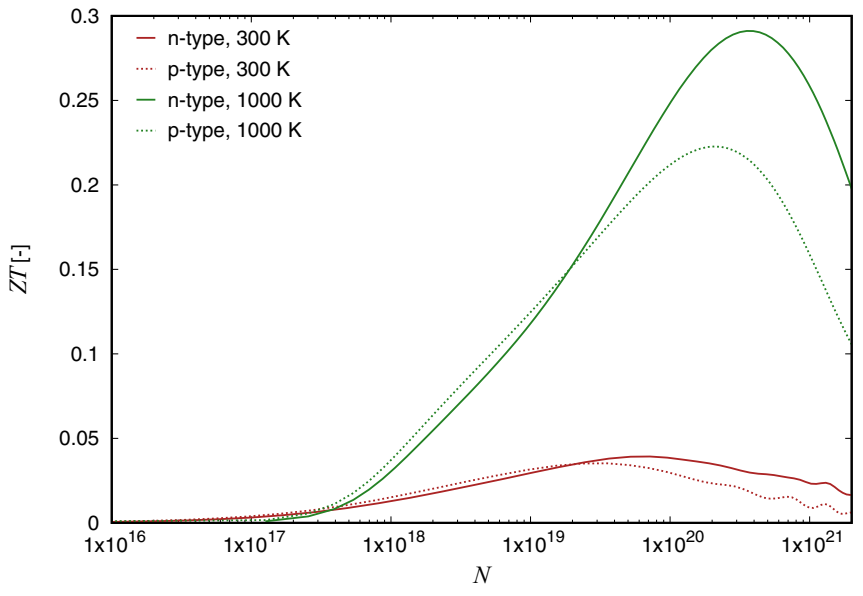

FIG. 8. Figure of merit $Z T$ in $\mathrm{Si}_{0.75} \mathrm{Ge}_{0.25}$ alloys as a function of the carrier concentration $N$ at $300 \mathrm{~K}$ (red lines) and $1000 \mathrm{~K}$ (green lines) with $n$-type (solid lines) and $p$-type (dotted lines) doping. already at low Ge content, while a further increase of the $\mathrm{Ge}$ content results in only marginal changes of $Z T$ in the $n$-type materials, as shown previously [13].

With the data discussed above, the thermoelectric figure of merit in bulk $\mathrm{Si}_{1-x} \mathrm{Ge}_{x}$ can be summarized as a function of carrier concentration, temperature, and alloy composition as shown in Fig. 9. The highest values are observed for heavily doped $\left(N=10^{20} \mathrm{~cm}^{-3}\right)$ Ge-rich $(x$ between 0.5 and $0.8)$ materials at high temperatures $(>1000 \mathrm{~K})$. The absolute values calculated here are lower than the commonly reported values around $0.8-1$ for SiGe alloys at elevated temperatures $[9,13,26]$. This difference is only due to extrinsic defects (not included here) largely affecting the thermal conductivity as discussed in the previous paragraph.

\section{CONCLUSIONS}

The thermoelectric properties described by the figure of merit $Z T$ of SiGe-based materials have been studied extensively in the literature. Optimization of $Z T$ as a result of the interdependent parameters, however, is not trivial. From an experimental point of view, a systematic study screening all material properties affecting the figure of merit is almost impossible since the accuracy of the alloy composition and carrier concentration is limited. Most computational studies 

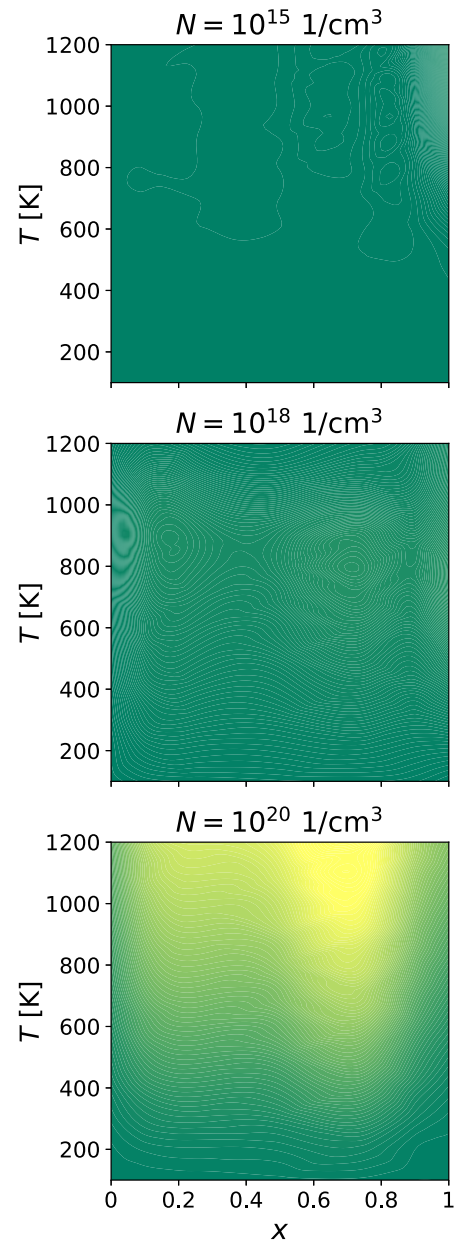
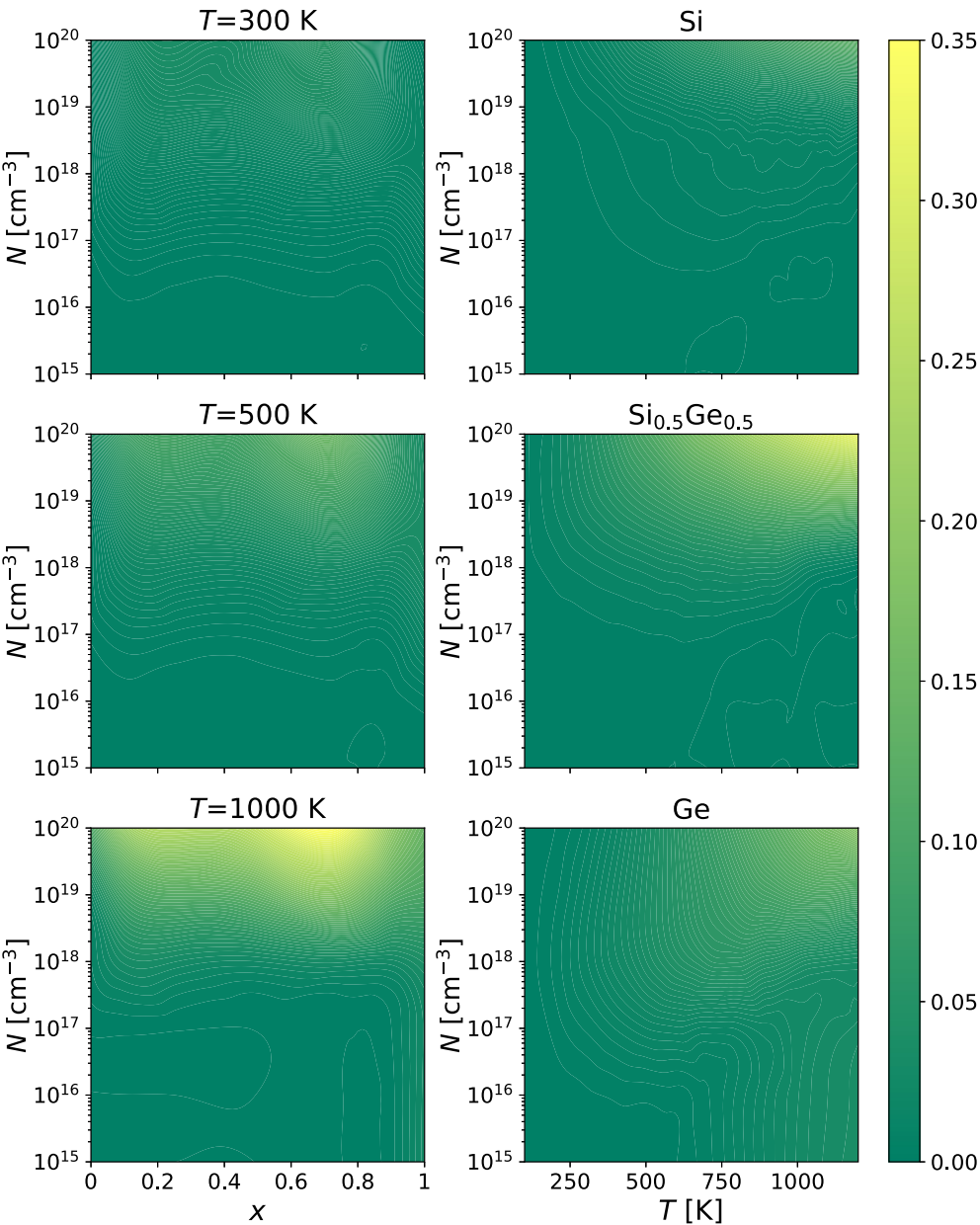

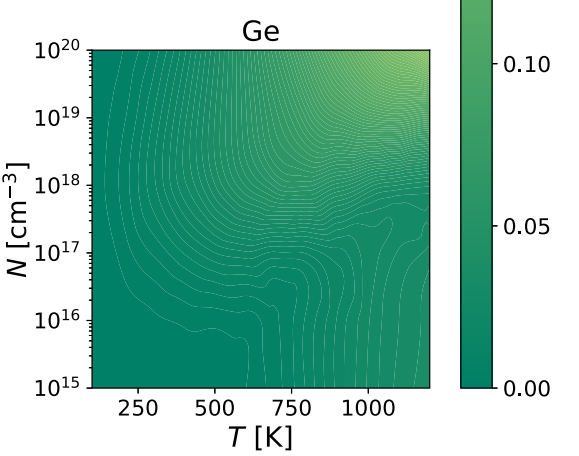

FIG. 9. Figure of merit of $n$-type $\mathrm{Si}_{1-x} \mathrm{Ge}_{x}$ alloys with varying carrier concentration $N$, Ge content $x$, and temperature $T$.

have focused either on the electronic properties or on the thermal transport relying on different methods for the two.

Here, we have carried out a consistent and systematic study based on first-principles calculations for both electronic and thermal transport properties in bulk SiGe alloys. The effect of alloy composition, carrier concentration, and temperature on the Seebeck coefficient, the electrical and thermal conductivity, and eventually the figure of merit has been evaluated. Seven different compositions of $\mathrm{Si}_{1-x} \mathrm{Ge}_{x}(x=0,0.1,0.25$, $0.5,0.75,0.9$, and 1) have been calculated, the carrier concentration has been changed from $10^{15}$ to $10^{20} \mathrm{~cm}^{-3}$, and temperatures from 100 to $1200 \mathrm{~K}$ have been considered.

The Seebeck coefficient is found to decrease exponentially with increasing carrier concentration, as expected. It is lowest in Ge-rich materials, and in general lower values are observed in $n$-type materials with respect to $p$-type. At intermediate carrier concentrations, it shows a maximum between 700 and $900 \mathrm{~K}$, which is lower in height and at lower temperatures for Ge-rich materials. In $p$-type materials, it steadily shifts to lower temperatures with increasing Ge content. Other than a small decrease for Ge-rich materials $(x \geqslant 0.9)$, the Seebeck coefficient hardly changes with varying alloy composition. Overall, it is found to be highest in both $n$ - and $p$-type materials for light doping $\left(10^{15}-10^{17} \mathrm{~cm}^{-3}\right)$ at intermediate temperatures (300-500 K) and in Si-rich materials.
At variance with the Seebeck coefficient, the electrical conductivity increases linearly with increasing carrier concentration. In $n$-type materials at room temperature it is highest in pure $\mathrm{Ge}$, whereas in p-type materials it is highest in $\mathrm{Si}_{0.25} \mathrm{Ge}_{0.75}$ for increased carrier concentrations $\left(N \geqslant 10^{19} \mathrm{~cm}^{-3}\right)$. At intermediate carrier concentrations, it has a minimum between 700 and $900 \mathrm{~K}$ in both $n$ - and $p$-type materials. Only marginal effects are observed with changing alloy composition except for pure Ge, where the electrical conductivity is found to be higher with respect to all other compositions. In summary, the electrical conductivity is highest for heavy doping $\left(>10^{19} \mathrm{~cm}^{-3}\right)$ and at intermediate temperatures $(<500 \mathrm{~K})$. At elevated temperatures, higher values are also observed for light doping in Ge-rich materials.

In agreement with numerous experimental and theoretical studies, the thermal conductivity is calculated to decrease remarkably with respect to pure $\mathrm{Si}$ and Ge already at small alloy compositions, $x$ and $1-x$, respectively. It is found to be higher compared to many other experimental studies resulting from the notable decrease in thermal conductivity already at small impurities and affected by the production method of the samples. Based on the theoretical framework used here (an ideal system with no boundaries and no lattice defects with periodic boundary conditions), the calculated values of this study can be considered as intrinsic property giving an upper 
limit of the thermal conductivity. The lattice thermal conductivity decreases with increasing temperature. This decrease is most pronounced in the pure $\mathrm{Si}$ and Ge materials.

The overall thermoelectric performance summarized in the intrinsic figure of merit $Z T$ in general increases with increasing carrier concentration and increasing temperature as a result of the increasing electrical conductivity and decreasing thermal conductivity. Depending on the carrier concentration and alloy composition, it shows a maximum for temperatures between 800 and $1200 \mathrm{~K}$. The maximum found with respect to the carrier concentration increases and shifts to higher carrier concentrations with increasing temperature. The ultimate highest values of $Z T$ are found for heavy doping $\left(>10^{19} \mathrm{~cm}^{-3}\right)$ and elevated temperatures $(>1000 \mathrm{~K})$ in $\mathrm{Si}_{1-x} \mathrm{Ge}_{x}$ alloys with Ge content from 0.5 to 0.8 in agreement with previous studies demonstrating the capability of firstprinciples calculations to adequately describe thermoelectric properties without the need of empirical parameters. This study paves the way to further improve the calculation of thermoelectric properties in realistic materials with any degree of structural complexity based on first-principles methods.

\section{ACKNOWLEDGMENTS}

We express our gratitude to Giorgia Fugallo (LTeN CNRS and Univerité de Nantes) for her generous help and fruitful discussions. K.R.H. also thanks G.F. for great hospitality. This work is financed by Ministero dell'Università e Ricerca (MIUR) under the Piano Operativo Nationale 2014-2020 asse I, action I.2 "Mobilità dei Ricercatori" (PON 20142020, AIM), through project AIM1809115-1. Simulations have partly been conducted on the HPC resources of CINECA under the project ISCRA_SINANOW.
[1] D. Beretta, N. Neophytou, J. M. Hodges, M. G. Kanatzidis, D. Narducci, M. Martin-Gonzalez, M. Beekman, B. Balke, G. Cerretti, W. Tremel, A. Zevalkink, A. I. Hofmann, C. Müller, B. Dörling, M. Campoy-Quiles, and M. Caironi, Mater. Sci. Eng., R 138, 100501 (2019).

[2] M. S. Dresselhaus, G. Chen, M. Y. Tang, R. G. Yang, H. Lee, D. Z. Wang, Z. F. Ren, J. P. Fleurial, and P. Gogna, Adv. Mater. 19, 1043 (2007).

[3] A. J. Minnich, M. S. Dresselhaus, Z. F. Ren, and G. Chen, Energy Environ. Sci. 2, 466 (2009).

[4] J. Karni, Nat. Mater. 10, 481 (2011).

[5] G. J. Snyder and E. S. Toberer, Nat. Mater. 7, 105 (2008).

[6] J. K. Yu, S. Mitrovic, D. Tham, J. Varghese, and J. R. Heath, Nat. Nanotechnol. 5, 718 (2010).

[7] A. V. Ioffe and A. F. Ioffe, Dokl. Akad. Nauk SSSR 8, 757 (1954).

[8] B. Abeles, Phys. Rev. 131, 1906 (1963).

[9] J. P. Dismukes, L. Ekstrom, E. F. Steigmeier, I. Kudman, and D. S. Beers, J. Appl. Phys. 35, 2899 (1964).

[10] G. A. Slack and M. A. Hussain, J. Appl. Phys. 70, 2694 (1991).

[11] A. N. Scoville, C. Bajgar, J. Vandersande, and J.-P. Fleurial, in 27th Intersociety Energy Conversion Engineering Conference (SAE International, San Diego, CA, 1992).

[12] I. O. Thomas and G. P. Srivastava, Phys. Rev. B 86, 045205 (2012).

[13] F. Murphy-Armando, J. Appl. Phys. 126, 215103 (2019).

[14] H. Stöhr and W. Klemm, Z. Anorg. Allg. Chem. 241, 305 (1939).

[15] X. W. Wang, H. Lee, Y. C. Lan, G. H. Zhu, G. Joshi, D. Z. Wang, J. Yang, A. J. Muto, M. Y. Tang, J. Klatsky, S. Song, M. S. Dresselhaus, G. Chen, and Z. F. Ren, Appl. Phys. Lett. 93, 193121 (2008).

[16] J. Garg, N. Bonini, B. Kozinsky, and N. Marzari, Phys. Rev. Lett. 106, 045901 (2011).

[17] C. Melis and L. Colombo, Phys. Rev. Lett. 112, 065901 (2014).

[18] A. J. Minnich, H. Lee, X. W. Wang, G. Joshi, M. S. Dresselhaus, Z. F. Ren, G. Chen, and D. Vashaee, Phys. Rev. B 80, 155327 (2009).
[19] Y. He, D. Donadio, and G. Galli, Appl. Phys. Lett. 98, 144101 (2011).

[20] E. K. Lee, L. Yin, Y. Lee, J. W. Lee, S. J. Lee, J. Lee, S. N. Cha, D. Whang, G. S. Hwang, K. Hippalgaonkar, A. Majumdar, C. Yu, B. L. Choi, J. M. Kim, and K. Kim, Nano Lett. 12, 2918 (2012).

[21] I. Savic, D. Donadio, F. Gygi, and G. Galli, Appl. Phys. Lett. 102, 073113 (2013).

[22] L. Ferre Llin, A. Samarelli, S. Cecchi, T. Etzelstorfer, E. Muller Gubler, D. Chrastina, G. Isella, J. Stangl, J. M. R. Weaver, P. S. Dobson, and D. J. Paul, Appl. Phys. Lett. 103, 143507 (2013).

[23] R. Rurali, X. Cartoixà, and L. Colombo, Phys. Rev. B 90, 041408(R) (2014).

[24] S. I. Yi and C. Yu, J. Appl. Phys. 117, 035105 (2015).

[25] H. R. Meddins and J. E. Parrott, J. Phys. C 9, 1263 (1976).

[26] C. B. Vining, J. Appl. Phys. 69, 331 (1991).

[27] L. Shi, D. Yao, G. Zhang, and B. Li, Appl. Phys. Lett. 95, 063102 (2009).

[28] L. Shi, J. Jiang, G. Zhang, and B. Li, Appl. Phys. Lett. 101, 233114 (2012).

[29] P. Garcia-Fernandez, M. Verissimo-Alves, D. I. Bilc, P. Ghosez, and J. Junquera, Phys. Rev. B 86, 085305 (2012).

[30] N. Miao, B. Xu, N. C. Bristowe, D. I. Bilc, M. J. Verstraete, and P. Ghosez, J. Phys. Chem. C 120, 9112 (2016).

[31] K. R. Hahn, E. Assaf, A. Portavoce, S. Bertaina, and A. Charaï, J. Phys. Chem. C 121, 26575 (2017).

[32] G. K. Madsen and D. J. Singh, Comput. Phys. Commun. 175, 67 (2006)

[33] N. Yang, G. Zhang, and B. Li, Nano Lett. 8, 276 (2008).

[34] L. Shi, D. Yao, G. Zhang, and B. Li, Appl. Phys. Lett. 96, 173108 (2010).

[35] J. Chen, G. Zhang, and B. Li, Appl. Phys. Lett. 95, 073117 (2009).

[36] G. Fugallo and L. Colombo, Phys. Scr. 93, 043002 (2018).

[37] G. Fugallo and L. Colombo, Phys. Scr. 93, 59501 (2018).

[38] L. J. Porter, J. F. Justo, and S. Yip, J. Appl. Phys. 82, 5378 (1997). 
[39] Y. P. He, D. Donadio, and G. Galli, Nano Lett. 11, 3608 (2011).

[40] Y. He, I. Savić, D. Donadio, and G. Galli, Phys. Chem. Chem. Phys. 14, 16209 (2012).

[41] F. Müller-Plathe, J. Chem. Phys. 106, 6082 (1997).

[42] P. K. Schelling, S. R. Phillpot, and P. Keblinski, Phys. Rev. B 65, 144306 (2002).

[43] K. R. Hahn, C. Melis, and L. Colombo, Eur. Phys. J. B 87, 150 (2014).

[44] K. R. Hahn, S. Cecchi, and L. Colombo, Appl. Phys. Lett. 108, 203102 (2016).

[45] L. Paulatto, F. Mauri, and M. Lazzeri, Phys. Rev. B 87, 214303 (2013).

[46] G. Fugallo, M. Lazzeri, L. Paulatto, and F. Mauri, Phys. Rev. B 88, 045430 (2013).

[47] See supplemental material at http://link.aps.org/supplemental/ 10.1103/PhysRevMaterials.5.065403 for all implementation details, results of $p$-type materials and additional supporting figures.

[48] P. Giannozzi, S. Baroni, N. Bonini, M. Calandra, R. Car, C. Cavazzoni, D. Ceresoli, G. L. Chiarotti, M. Cococcioni, I. Dabo, A. Dal Corso, S. de Gironcoli, S. Fabris, G. Fratesi, R. Gebauer, U. Gerstmann, C. Gougoussis, A. Kokalj, M. Lazzeri, L. Martin-Samos et al., J. Phys.: Condens. Matter 21, 395502 (2009).

[49] W. Kohn and L. J. Sham, Phys. Rev. 140, A1133 (1965).

[50] N. Troullier and J. L. Martins, Phys. Rev. B 43, 1993 (1991).

[51] H. J. Monkhorst and J. D. Pack, Phys. Rev. B 13, 5188 (1976).

[52] M. Podgorny, G. Wolfgarten, and J. Pollmann, J. Phys. C 19, L141 (1986).

[53] S. de Gironcoli, P. Giannozzi, and S. Baroni, Phys. Rev. Lett. 66, 2116 (1991).

[54] N. Marzari, S. de Gironcoli, and S. Baroni, Phys. Rev. Lett. 72, 4001 (1994).

[55] D. M. Caughey and R. E. Thomas, Proc. IEEE 55, 2192 (1967).

[56] M. Glicksman, Phys. Rev. 111, 125 (1958).

[57] R. Braunstein, A. R. Moore, and F. Herman, Phys. Rev. 109, 695 (1958)

[58] A. Debernardi and S. Baroni, Solid State Commun. 91, 813 (1994).

[59] A. Debernardi, S. Baroni, and E. Molinari, Phys. Rev. Lett. 75, 1819 (1995).

[60] A. Cepellotti, G. Fugallo, L. Paulatto, M. Lazzeri, F. Mauri, and N. Marzari, Nat. Commun. 6, 6400 (2015).

[61] G. Fugallo, A. Cepellotti, L. Paulatto, M. Lazzeri, N. Marzari, and F. Mauri, Nano Lett. 14, 6109 (2014).

[62] M. Lazzeri, M. Calandra, and F. Mauri, Phys. Rev. B 68, 220509(R) (2003).
[63] J. Callaway, Phys. Rev. 113, 1046 (1959).

[64] P. G. Klemens, in Solid State Physics-Advances in Research and Applications, edited by F. Seitz and D. Turnbull (Academic, New York, 1958), Vol. 7, pp. 1-98.

[65] J. Ziman, Electrons and Phonons (Oxford University Press, Oxford, 2001).

[66] R. A. Guyer and J. A. Krumhansl, Phys. Rev. 148, 766 (1966).

[67] H. Lee, D. Vashaee, D. Z. Wang, M. S. Dresselhaus, Z. F. Ren, and G. Chen, J. Appl. Phys. 107, 094308 (2010).

[68] F. Murphy-Armando and S. Fahy, Phys. Rev. Lett. 97, 096606 (2006).

[69] G. Busch and O. Vogt, Helv. Phys. Acta 33, 437 (1960).

[70] A. Amith, Phys. Rev. 139, A1624 (1965).

[71] G. Joshi, H. Lee, Y. C. Lan, X. W. Wang, G. H. Zhu, D. Z. Wang, R. W. Gould, D. C. Cuff, M. Y. Tang, M. S. Dresselhaus, G. Chen, and Z. F. Ren, Nano Lett. 8, 4670 (2008).

[72] Z. Tian, J. Garg, K. Esfarjani, T. Shiga, J. Shiomi, and G. Chen, Phys. Rev. B 85, 184303 (2012).

[73] W. Li, L. Lindsay, D. A. Broido, D. A. Stewart, and N. Mingo, Phys. Rev. B 86, 174307 (2012).

[74] M. Arrigoni, J. Carrete, N. Mingo, and G. K. H. Madsen, Phys. Rev. B 98, 115205 (2018).

[75] S. Thébaud, C. A. Polanco, L. Lindsay, and T. Berlijn, Phys. Rev. B 102, 094206 (2020).

[76] B. Liao, B. Qiu, J. Zhou, S. Huberman, K. Esfarjani, and G. Chen, Phys. Rev. Lett. 114, 115901 (2015).

[77] C. J. Glassbrenner and G. A. Slack, Phys. Rev. 134, A1058 (1964).

[78] A. V. Inyushkin, A. N. Taldenkov, A. V. Gusev, A. M. Gibin, V. A. Gavva, and E. A. Kozyrev, Phys. Solid State 55, 235 (2013).

[79] V. I. Ozhogin, A. V. Inyushkin, A. N. Taldenkov, A. V. Tikhomirov, G. É. Popov, E. Haller, and K. Itoh, J. Exp. Theor. Phys. Lett. 63, 490 (1996).

[80] M. C. Steele and F. D. Rosi, J. Appl. Phys. 29, 1517 (1958).

[81] B. Abeles, D. S. Beers, G. D. Cody, and J. P. Dismukes, Phys. Rev. 125, 44 (1962).

[82] Y. Lee, A. J. Pak, and G. S. Hwang, Phys. Chem. Chem. Phys. 18, 19544 (2016).

[83] G. A. Slack, Phys. Rev. 105, 829 (1957).

[84] N. A. Katcho, J. Carrete, W. Li, and N. Mingo, Phys. Rev. B 90, 094117 (2014).

[85] C. A. Polanco and L. Lindsay, Phys. Rev. B 98, 014306 (2018).

[86] M. B. Maccioni, R. Farris, and V. Fiorentini, Phys. Rev. B 98, 220301(R) (2018).

[87] A. Skye and P. K. Schelling, J. Appl. Phys. 103, 113524 (2008). 